\title{
Existence and uniqueness results for $\Phi$-Caputo implicit fractional pantograph differential equation with generalized anti-periodic boundary condition
}

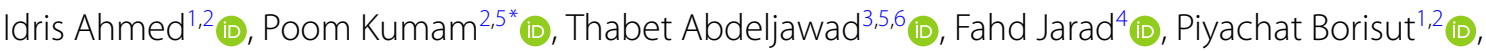 \\ Musa Ahmed Demba ${ }^{1,2}$ (I) and Wiyada Kumam ${ }^{7 *}$ (D)
}

\section{"Correspondence:}

poom.kum@kmuttac.th:

wiyada.kum@rmutt.ac.th

${ }^{2}$ Fixed Point Research Laboratory,

Fixed Point Theory and Applications

Research Group, Center of

Excellence in Theoretical and

Computational Science (TaCS-CoE),

Faculty of Science, King Mongkut's

University of Technology Thonburi

(KMUTT), 126 Pracha-Uthit Road,

Bang Mod, Thung Khru, Bangkok

10140, Thailand

${ }^{7}$ Program in Applied Statistics,

Department of Mathematics and

Computer Science, Rajamangala

University of Technology

Thanyaburi, Thanyaburi,

Pathumthani 12110, Thailand

Full list of author information is

available at the end of the article

\begin{abstract}
The present paper describes the implicit fractional pantograph differential equation in the context of generalized fractional derivative and anti-periodic conditions. We formulated the Green's function of the proposed problems. With the aid of a Green's function, we obtain an analogous integral equation of the proposed problems and demonstrate the existence and uniqueness of solutions using the techniques of the Schaefer and Banach fixed point theorems. Besides, some special cases that show the proposed problems extend the current ones in the literature are presented. Finally, two examples were given as an application to illustrate the results obtained.
\end{abstract}

MSC: $34 \mathrm{~K} 20 ; 34 \mathrm{~K} 37 ; 32 \mathrm{~F} 18$

Keywords: Pantograph differential equation; $\Phi$-Caputo fractional derivative; Anti-periodic condition

\section{Introduction}

The fractional calculus, which allows for the integration and differentiation of functions with non-integer orders, is one of the fastest-growing areas of mathematics based on the findings that fractional operators were used in mathematical modeling [10-12, 21, 25, $27,29]$. In [9, 19], the authors presented the so-called fractional differential operator of a function with respect to another function. These operators have generalized several wellknown fractional operators dealing with the fractional derivatives of Caputo, CaputoHadamard, Caputo-Erdélyi-Kober and Caputo-Katugampola. Also, these operators have been successfully used to solve population growth and other models.

The most favored area of study in the field of fractional differential equations, which has received significant attention from researchers, is the theory of existence and uniqueness of solutions. Many researchers have introduced some interesting results on the existence and uniqueness of solutions of various initial/boundary value problems, using different techniques used in fixed point theory. For more details we refer the reader to

(c) The Author(s) 2020. This article is licensed under a Creative Commons Attribution 4.0 International License, which permits use sharing, adaptation, distribution and reproduction in any medium or format, as long as you give appropriate credit to the original author(s) and the source, provide a link to the Creative Commons licence, and indicate if changes were made. The images or other third party material in this article are included in the article's Creative Commons licence, unless indicated otherwise in a credit line to the material. If material is not included in the article's Creative Commons licence and your intended use is not permitted by statutory regulation or exceeds the permitted use, you will need to obtain permission directly from the copyright holder. To view a copy of this licence, visit http://creativecommons.org/licenses/by/4.0/. 
$[5,6,16,18,26,30,31]$. In particular, the pantograph delay equation was used as an effective tool to gain insight into some of the current issues emerging from different fields of knowledge, such as quantum mechanics, probability, number theory, control systems and electrodynamics. However, a significant study has been carried out on the properties of this form of fractional differential equation, both analytical and numerical, and interesting results have been published; see $[13,15,23]$.

Recently, Idris et al. [7] examined the implicit fractional pantograph differential equation given by

$$
\left\{\begin{array}{l}
{ }^{H} \mathbb{D}_{0^{+}}^{\gamma, p ; \phi} u(t)=g\left(t, u(t), u(\vartheta t),{ }^{H} \mathbb{D}_{0^{+}}^{\gamma, p ; \phi} u(\vartheta t)\right), \quad t \in(0, b], 0<\gamma<1,0<\vartheta<1, \\
\mathbb{I}_{0^{+}}^{1-\beta ; \phi} u\left(0^{+}\right)=\sum_{k=1}^{n} d_{k} \mathbb{I}_{0^{+}}^{q ; \phi} u\left(\iota_{k}\right), \quad \beta=\gamma+p-\gamma p, 0<\iota_{k}<b, d_{k} \in \mathbb{R}, n \in \mathbb{N},
\end{array}\right.
$$

where ${ }^{H} \mathcal{D}_{0^{+}}^{\gamma, p ; \phi}(\cdot)$ represent the fractional derivative of order $\gamma$ and parameter $(0 \leq p \leq 1)$, in $\phi$-Hilfer setting. $\mathbb{I}_{0^{+}}^{1-\beta ; \phi}(\cdot)$ and $\mathbb{I}_{0^{+}}^{q ; \phi}(\cdot)$ are $\phi$-Riemann-Liouville fractional integrals of order $1-\beta$ and $q>0$, respectively and $g:(0, b] \times \mathbb{R}^{3} \rightarrow \mathbb{R}$ is a continuous function. The authors have established the existence and uniqueness of solutions using Schaefer's and Banach's fixed point theorem techniques. Besides, two different kinds of stability were discussed in the context of Ulam-Hyers and the generalized Ulam-Hyers theory. Antiperiod problems have attracted the attention of researchers as anti-period boundary conditions occur in different disciplines. In the last two decades, these problems have been utilized for a wide variety of fields of science and engineering, such as underground water flow, populations dynamics and blood flow. Literature related to these problems can be found in $[4,28,32,34,35]$.

Li et al. [22] studied the impulsive boundary value problem described by

$$
\left\{\begin{array}{l}
{ }^{C} D_{0^{+}}^{\rho} v(t)=\mathcal{F}(t, v(t)), \quad t \in \mathcal{J}^{*}=[0,1] \backslash\left\{t_{1}, \ldots, t_{k}\right\} \\
\Delta v\left(t_{j}\right)=I_{j}, \quad \Delta v^{\prime}\left(t_{j}\right)=J_{j}, \quad j=1, \ldots, k \\
c_{1} v(0)=-c_{2} v(1), \quad c_{1} v^{\prime}(0)=-c_{2} v^{\prime}(1)
\end{array}\right.
$$

where ${ }^{C} D_{0^{+}}^{\rho}(\cdot)$ is the Caputo fractional derivative of order $(1<\rho<2), c_{1} \geq c_{2}>0, \mathcal{F}$ : $[0,1] \times \mathbb{R} \rightarrow \mathbb{R}$ is jointly continuous function, $I_{j}, J_{j} \in \mathbb{R}$ and $t_{j}$ satisfy $0=t_{0}<t_{1}<\cdots<$ $t_{k}<t_{k+1}=1, \Delta v\left(t_{j}\right)=v\left(t_{j}^{+}\right)-v\left(t_{j}^{-}\right)$with $v\left(t_{j}^{+}\right)=\lim _{\epsilon \rightarrow 0^{+}} v\left(t_{j}+\epsilon\right)$ and $v\left(t_{j}^{-}\right)=\lim _{\epsilon \rightarrow 0^{-}} v\left(t_{j}+\epsilon\right)$ representing the right and left limits of $v(t)$ at $t=t_{j}$. Recently, Ali et al. [8] examined the existence, uniqueness and stability of the boundary value problem:

$$
\left\{\begin{array}{l}
{ }^{C} \mathrm{D}_{0^{+}}^{\alpha} w(t)=\Theta\left(t, w(t), w(\zeta t),{ }^{C} \mathrm{D}_{0^{+}}^{\alpha} w(t)\right), \\
\quad t \in[0, \mathfrak{T}], 2<\alpha \leq 3,0<\zeta<1, \mathfrak{T}>0, \\
w(0)=-w(\mathfrak{T}), \quad{ }^{C} \mathrm{D}_{0^{+}}^{r} w(0)=-{ }^{C} \mathrm{D}_{0^{+}}^{r} w(\mathfrak{T}), \quad{ }^{C} \mathrm{D}_{0^{+}}^{\tau} w(0)=-{ }^{C} \mathrm{D}_{0^{+}}^{\tau} w(\mathfrak{T}),
\end{array}\right.
$$

where ${ }^{C} \mathrm{D}_{0^{+}}^{\alpha}(\cdot),{ }^{C} \mathrm{D}_{0^{+}}^{r}(\cdot),{ }^{C} \mathrm{D}_{0^{+}}^{\tau}(\cdot)$ are Caputo fractional derivatives of order $(2<\alpha \leq 3),(0<$ $r \leq 1)$ and $(1<\tau \leq 2)$, respectively, and $\Theta \in \mathcal{C}\left([0, \mathfrak{T}], \mathbb{R}^{3}, \mathbb{R}\right)$.

Inspired by the above recent results, we investigate the existence and uniqueness of solutions of the following nonlinear implicit fractional pantograph differential equation with 
$\Phi$-Caputo fractional derivative and more general anti-periodic boundary condition (IFPBVPs). In short the IFPBVPs are of the form

$$
\left\{\begin{array}{l}
{ }^{C} \mathcal{D}_{0^{+}}^{r ; \Phi} z(t)=f\left(t, z(t), z(\sigma t),{ }^{C} \mathcal{D}_{0^{+}}^{r ; \Phi} z(t)\right), \quad t \in \mathcal{J}=[0, \xi], 0<\sigma<1, \xi>0, \\
\alpha_{1} z(0)=-\alpha_{2} z(\xi), \quad \alpha_{1}{ }^{C} \mathcal{D}_{0^{+}}^{p ; \Phi} z(0)=-\alpha_{2}{ }^{C} \mathcal{D}_{0^{+}}^{p ; \Phi} z(\xi), \\
\alpha_{1}{ }^{C} \mathcal{D}_{0^{+}}^{q ; \Phi} z(0)=-\alpha_{2}{ }^{C} \mathcal{D}_{0^{+}}^{q ; \Phi} z(\xi),
\end{array}\right.
$$

where ${ }^{C} \mathcal{D}_{0^{+}}^{r ; \Phi}(\cdot),{ }^{C} \mathcal{D}_{0^{+}}^{p ; \Phi}(\cdot)$ and ${ }^{C} \mathcal{D}_{0^{+}}^{q ; \Phi}(\cdot)$ are $\Phi$-Caputo fractional derivative of order $(2<$ $r \leq 3),(0<p \leq 1)$ and $(1<q \leq 2)$, respectively with respect to $\Phi \in \mathcal{C}([\mathcal{J}, \mathbb{R}])$ such that $\Phi^{\prime}(t)>0$, for all $t \in \mathcal{J}, \alpha_{1} \geq \alpha_{2}>0$.

Moreover, we also study the nonlinear implicit fractional pantograph boundary value problems (IFPBVPs) of the form

$$
\left\{\begin{array}{l}
{ }^{C} \mathcal{D}_{0^{+}}^{r ; \Phi} z(t)=f\left(t, z(t), z(\sigma t),{ }^{C} \mathcal{D}_{0^{+}}^{r ; \Phi} z(t)\right), \quad t \in \mathcal{J}=[0, \xi], 0<\sigma<1, \xi>0, \\
\alpha_{1} z(0)+\alpha_{2} z(\xi)=0, \quad \alpha_{1} \delta_{\Phi} z(0)+\alpha_{2} \delta_{\Phi} z(\xi)=0,
\end{array}\right.
$$

where ${ }^{C} \mathcal{D}_{0^{+}}^{r ; \Phi}(\cdot)$ is a $\Phi$-Caputo fractional derivative of order $(1<r \leq 2)$ with respect to another function $\Phi \in \mathcal{C}([\mathcal{J}, \mathbb{R}])$ such that $\Phi^{\prime}(t)>0$, for all $t \in \mathcal{J}, \alpha_{1} \geq \alpha_{2}>0, \delta_{\Phi}=\left(\frac{1}{\Phi^{\prime}(t)} \frac{d}{d t}\right) z(t)$ and $f:[0, \xi] \times \mathbb{R}^{3} \rightarrow \mathbb{R}$ is a given continuous function.

\section{Remark 1.1}

i. Observe that, if $\alpha_{1}=\alpha_{2}=1$ and $\Phi(t)=t$, problems (1.4) and coincide with Caputo fractional differential equation considered in $[8]$ and $[1,3,14]$ respectively.

ii. If $\Phi(t)=\ln t$ problems (1.4) and (1.5) reduce to the Caputo-Hadamard fractional differential equation [17].

iii. If $\Phi(t)=t^{\sigma}$ problems (1.4) and (1.5) reduce to the Caputo-Erdélyi-Kober fractional differential equation [24].

iv. If $\Phi(t)=\frac{t^{\sigma}}{\sigma}, \sigma>0$ problems (1.4) and (1.5) reduce to the Caputo-Katugampola fractional differential equation [20].

To the best of our knowledge, this is the first paper to discuss the existence and uniqueness of solutions for a class of nonlinear implicit fractional pantograph differential equations using $\Phi$-Caputo fractional derivative with a more general anti-periodic boundary condition. Also, this work contributes to an improvement in the qualitative aspects of fractional calculus, boundary value problems, pantograph equations. However, the $\Phi$-Caputo fractional and anti-periodic condition considered in this work is more general than the current ones described in the literature (see the Conclusion section for more details).

The outlined of this paper is as follows: In Sect. 2, we recall the main definitions, the $\Phi$-Caputo fractional derivative and some theoretical results in line with the $\Phi$-fractional operators needed in a later section. In Sect. 3, we establish a relation between the proposed problems and mixed-type integral equation with the help of a Green's function. Besides, we investigate the existence and uniqueness of solutions using the techniques of Schaefer's and Banach's fixed point theorems. Some particular cases and theoretical examples are discussed, as can be seen in this section. Finally, we summarize the theoretical results in Sect. 4. 


\section{Preliminaries}

This section recalled some basic definitions and lemmas concerning fractional operators that are essential throughout the writing of the paper.

Let $\mathbb{E}=\mathcal{C}([\mathcal{J}, \mathbb{R}])$ be a Banach space equipped with the norm defined by

$$
\|z\|_{\mathbb{E}}=\max \{|z(t)|: t \in \mathcal{J}\} .
$$

The space of all absolutely continuous real valued function on $\mathcal{J}$ is denoted by $\mathcal{A C}([\mathcal{J}, \mathbb{R}])$. So, we define the space $\mathcal{A C}_{\Phi}^{n}([\mathcal{J}, \mathbb{R}])$ by

$$
\mathcal{A C}_{\Phi}^{n}([\mathcal{J}, \mathbb{R}])=\left\{f: \mathcal{J} \rightarrow \mathbb{R} ;\left(\delta_{\Phi}^{n-1} f\right)(t) \in \mathcal{A C}([\mathcal{J}, \mathbb{R}]), \delta_{\Phi}=\frac{1}{\Phi^{\prime}(t)} \frac{d}{d t}\right\}
$$

with the norm defined by

$$
\|f\|_{\mathcal{A} \mathcal{C}_{\Phi}^{n}}=\sum_{k=0}^{n-1}\left\|\delta_{\Phi}^{k} f\right\|_{\mathbb{E}}
$$

where $\Phi \in \mathcal{C}^{n}([\mathcal{J}, \mathbb{R}]), \Phi^{\prime}(t)>0$ on $\mathcal{J}$ and $\delta_{\Phi}^{k}=\underbrace{\delta_{\Phi} \delta_{\Phi} \cdots \delta_{\Phi}}_{\text {k-times }}$.

Definition 2.1 ([21]) Let $f \in L^{1}[a, b]$ be a function. Then the fractional operator

$$
\mathcal{I}_{a}^{r} f(t)=\frac{1}{\Gamma(r)} \int_{a}^{t}(t-\tau)^{r-1} f(\tau) d \tau, \quad r>0, t>a \geq 0,
$$

is referred to as a Riemann-Liouville integral of order $r$ provided the right-hand side of (2.1) exists.

Definition 2.2 ([21]) Let $n \in \mathbb{N}, r, t, a \in \mathbb{R}_{+}$and the function $f \in \mathcal{C}^{n}[a, b]$. Then the fractional operator

$$
{ }^{C} \mathcal{D}_{a}^{r} f(t)=\left\{\begin{array}{l}
\frac{1}{\Gamma(n-r)} \int_{a}^{t}(t-\tau)^{n-r-1} f^{n}(\tau) d \tau, \quad r>0, t>a \geq 0, \\
\frac{d^{n}}{d t^{n}} z(t), \quad r=n,
\end{array}\right.
$$

is referred to as Caputo's fractional derivative of order $r$ provided that the right-hand side of $(2.2)$ is point-wise defined on $(a, \infty)$ and $n=[r]+1$.

Definition 2.3 ([21]) Suppose $(0, b] \subset \mathbb{R}_{+}$is a finite or infinite interval. Let $f \in L^{1}[0, b]$ and $\Phi(t)>0$ be monotone function on $(0, b]$ such that $\Phi^{\prime}(t) \in C([(0, b), \mathbb{R}])$. Then the fractional operator

$$
\left(\mathcal{I}_{0^{+}}^{r ; \Phi} f\right)(t)=\frac{1}{\Gamma(r)} \int_{0}^{t} \Phi^{\prime}(s)(\Phi(t)-\Phi(\tau))^{r-1} f(\tau) d \tau, \quad r>0, t>0,
$$

is called a $\Phi$-Riemann-Liouville fractional integral of order $r$ of the function $f$ with respect to another function $\Phi$. 
Definition 2.4 ([9, 19]) Let $f, \Phi$ be two function such that $f \in \mathcal{A C}_{\Phi}^{n}([\mathcal{J}, \mathbb{R}]), \Phi \in$ $\mathcal{C}^{n}([\mathcal{J}, \mathbb{R}]), \Phi(t)>0$ and $\Phi^{\prime}(t) \neq 0$ for all $t \in \mathcal{J}$. Then the fractional operator

$$
{ }^{C} \mathcal{D}_{0^{+}}^{r ; \Phi} f(t)=\left\{\begin{array}{l}
\mathcal{I}_{0^{+}}^{(n-r) ; \Phi}\left(\delta_{\Phi}^{n} f\right)(t), \quad r>0, n=[r]+1, n \in \mathbb{N} \\
\left(\delta_{\Phi}^{n} f\right)(t), \quad r=n \in \mathbb{N}
\end{array}\right.
$$

is referred to as the the left-sided $\Phi$-Caputo fractional derivative of a function $f$ of order $r$ with respect to another function $\Phi$.

Lemma 2.5 ([9]) Given $f(t)=(\Phi(t)-\Phi(0))^{k}$ and $r>0$. Then we have

$$
{ }^{C} \mathcal{D}_{0^{+}}^{r ; \Phi} f(t)= \begin{cases}\frac{\Gamma(k)}{\Gamma(k-r)}(\Phi(t)-\Phi(0))^{k-r-1}, & k \in \mathbb{R}, k>n, \\ \frac{k !}{\Gamma(k+1-r)}(\Phi(t)-\Phi(0))^{k-r}, & n \leq k \in \mathbb{N}, \\ 0, & n>k \in \mathbb{N} \cup\{0\} .\end{cases}
$$

Lemma 2.6 ([19]) Suppose $f \in \mathcal{A C}_{\Phi}^{n}([\mathcal{J}, \mathbb{R}])$ and $n-1<r \leq n, n \in \mathbb{N}$. Then

$$
\mathcal{I}_{0^{+}}^{r ; \Phi} \mathcal{D}_{0^{+}}^{r ; \Phi} f(t)=f(t)-\sum_{k=0}^{n-1} \frac{(\Phi(t)-\Phi(0))^{k}}{k !}\left(\delta_{\Phi}^{n} f\right)(0)
$$

for all $t \in \mathcal{J}$. Moreover, it for $2<r \leq 3$ yields

$$
\mathcal{I}_{0^{+}}^{r ; \Phi} \mathcal{D}_{0^{+}}^{r, \Phi} f(t)=f(t)-b_{0}-b_{1}(\Phi(t)-\Phi(0))-b_{2}(\Phi(t)-\Phi(0))^{2},
$$

where $b_{0}, b_{1}, b_{2}$ are arbitrary constants on $\mathbb{R}$.

Theorem 2.7 (Schaefer's fixed point theorem [36]) Let $\mathcal{N}: \mathbb{E} \rightarrow \mathbb{E}$ be a completely continuous operator. Suppose that the set $\vartheta(\mathcal{N})=\{z \in \mathbb{E}: z=\epsilon(\mathcal{N} z)$, for some $\epsilon \in[0,1]\}$ is bounded, then $\mathcal{N}$ has a fixed point.

Theorem 2.8 (Banach's fixed point theorem [36]) Let $\mathbb{E}$ be a complete metric space, and $\mathcal{N}$ be a contraction on $\mathbb{E}$. Then there exists a unique $z \in \mathbb{E}$ such that $\mathcal{N}(z)=z$.

\section{Main results}

In this section, we transform the proposed problem (1.4) into an equivalent integral equation with the help of the Green's function. Besides, the existence and uniqueness of solutions of problem (1.4) were establish using the techniques of Schaefer's and Banach's fixed point theorems.

Lemma 3.1 Suppose that $2<r \leq 3$ and $f: \mathcal{J} \times \mathbb{R}^{3} \rightarrow \mathbb{R}$ be a continuous function for any $z \in \mathcal{A C}_{\Phi}^{2}([\mathcal{J}, \mathbb{R}])$. A function $z \in \mathcal{A C}_{\Phi}^{2}([\mathcal{J}, \mathbb{R}])$ is a solution of the problem $(1.4)$ if and only if $z$ satisfies the following integral equation:

$$
z(t)=\int_{0}^{\xi} \mathcal{H}(t, \tau) \Phi^{\prime}(\tau) f\left(\tau, z(\tau), z(\sigma \tau),{ }^{C} \mathcal{D}_{0^{+}}^{r ; \Phi} z(\tau)\right) d \tau,
$$


where $\mathcal{H}(t, \tau)$ is the Green's function described by

$$
\begin{aligned}
& \mathcal{H}(t, \tau) \\
& =\left\{\begin{array}{c}
\frac{\left(\alpha_{1}+\alpha_{2}\right)(\Phi(t)-\Phi(\tau))^{r-1}-\alpha_{2}(\Phi(\xi)-\Phi(\tau))^{r-1}}{\left(\alpha_{1}+\alpha_{2}\right) \Gamma(r)} \\
\quad+\frac{\Gamma(2-p)\left[\alpha_{2}(\Phi(\xi)-\Phi(0))-\left(\alpha_{1}+\alpha_{2}\right)(\Phi(t)-\Phi(0))\right]}{\left(\alpha_{1}+\alpha_{2}\right) \Gamma(r-p)(\Phi(\xi)-\Phi(0))^{1-p}}(\Phi(\xi)-\Phi(\tau))^{r-p-1} \\
-\left[\frac{\Gamma(3-q)\left[p \alpha_{2}(\Phi(\xi)-\Phi(0))^{2}-2\left(\alpha_{1}+\alpha_{2}\right)(\Phi(\xi)-\Phi(0))+\left(\alpha_{1}+\alpha_{2}\right)(2-p)(\Phi(t)-\Phi(0))^{2}\right]}{2\left(\alpha_{1}+\alpha_{2}\right)(2-p) \Gamma(r-q)(\Phi(\xi)-\Phi(0))^{1-p}}\right] \\
\quad \times(\Phi(\xi)-\Phi(\tau))^{r-q-1}, \quad 0 \leq \tau \leq t \leq \xi ; \\
\frac{\alpha_{2}(\Phi(\xi)-\Phi(0))^{r-1}}{\left(\alpha_{1}+\alpha_{2}\right) \Gamma(r)}+\frac{\Gamma(2-p)\left[\alpha_{2}(\Phi(\xi)-\Phi(0))-\left(\alpha_{1}+\alpha_{2}\right)(\Phi(t)-\Phi(0))\right]}{\left(\alpha_{1}+\alpha_{2}\right) \Gamma(r-p)(\Phi(\xi)-\Phi(0))^{1-p}}(\Phi(\xi)-\Phi(\tau))^{r-p-1} \\
-\left[\frac{\Gamma(3-q)\left[p \alpha_{2}(\Phi(\xi)-\Phi(0))^{2}-2\left(\alpha_{1}+\alpha_{2}\right)(\Phi(\xi)-\Phi(0))(\Phi(t)-\Phi(0))+\left(\alpha_{1}+\alpha_{2}\right)(2-p)(\Phi(t)-\Phi(0))^{2}\right]}{2\left(\alpha_{1}+\alpha_{2}\right)(2-p) \Gamma(r-q)(\Phi(\xi)-\Phi(0))^{1-p}}\right] \\
\quad \times(\Phi(\xi)-\Phi(\tau))^{r-q-1}, \quad 0 \leq t \leq \tau \leq \xi .
\end{array}\right.
\end{aligned}
$$

For the sake of simplicity, we will denote $\mathcal{T}_{z}(t)={ }^{C} \mathcal{D}_{0^{+}}^{r ; \Phi} z(t)=f\left(t, z(t), z(\sigma t), \mathcal{T}_{z}(t)\right), K_{\Phi}(\xi)=$ $(\Phi(\xi)-\Phi(0))$ and $K_{\Phi}(t)=(\Phi(t)-\Phi(0))$.

Proof Suppose $z \in \mathcal{A C}_{\Phi}^{2}([\mathcal{J}, \mathbb{R}])$ satisfies problem (1.4), then it is enough to show that $z$ also satisfies the integral equation (3.1). Indeed, by applying the $\Phi$-Riemann-Liouville fractional operator to both sides of the first equation of problem (1.4) and making use of Lemma 2.6 yield

$$
\begin{aligned}
z(t)= & \frac{1}{\Gamma(r)} \int_{0}^{t} \Phi^{\prime}(\tau)(\Phi(t)-\Phi(\tau))^{r-1} \mathcal{T}_{z}(\tau) d \tau \\
& -b_{0}-b_{1}(\Phi(t)-\Phi(0))-b_{2}(\Phi(t)-\Phi(0))^{2} \\
= & \mathcal{I}_{0^{+}}^{r ; \Phi} \mathcal{T}_{z}(t)-b_{0}-b_{1}(\Phi(t)-\Phi(0))-b_{2}(\Phi(t)-\Phi(0))^{2}
\end{aligned}
$$

where $b_{0}, b_{1}, b_{2} \in \mathbb{R}$, are arbitrary constants to be determine. Using the facts that ${ }^{C} \mathcal{D}_{0^{+}}^{p ; \Phi} c=$ 0 (c is constant), ${ }^{C} \mathcal{D}_{0^{+}}^{p ; \Phi}(\Phi(t)-\Phi(0))=\frac{(\Phi(t)-\Phi(\tau))^{1-p}}{\Gamma(2-p)},{ }^{C} \mathcal{D}_{0^{+}}^{p ; \Phi}(\Phi(t)-\Phi(0))^{2}=\frac{2(\Phi(t)-\Phi(\tau))^{2-p}}{\Gamma(3-p)}$ and ${ }^{C} \mathcal{D}_{0^{+}}^{p ; \Phi} \mathcal{I}_{0^{+}}^{r ; \Phi} \mathcal{T}_{z}(t)=\mathcal{I}_{0^{+}}^{r-p ; \Phi} \mathcal{T}_{z}(t)$, we get

$$
{ }^{C} \mathcal{D}_{0^{+}}^{p ; \Phi} z(t)=\mathcal{I}_{0^{+}}^{r-p ; \Phi} \mathcal{T}_{z}(t)-\frac{b_{1}(\Phi(t)-\Phi(\tau))^{1-p}}{\Gamma(2-p)}-\frac{2 b_{2}(\Phi(t)-\Phi(\tau))^{2-p}}{\Gamma(3-p)}
$$

Similarly, ${ }^{C} \mathcal{D}_{0^{+}}^{q ; \Phi}(\Phi(t)-\Phi(0))=0(1<q \leq 2),{ }^{C} \mathcal{D}_{0^{+}}^{q ; \Phi}(\Phi(t)-\Phi(0))^{2}=\frac{2(\Phi(t)-\Phi(\tau))^{2-q}}{\Gamma(3-q)}$ and ${ }^{C} \mathcal{D}_{0^{+}}^{q ; \Phi} \mathcal{I}_{0^{+}}^{r, \Phi} \mathcal{T}_{z}(t)=\mathcal{I}_{0^{+}}^{r-q ; \Phi} \mathcal{T}_{z}(t)$ give

$$
{ }^{C} \mathcal{D}_{0^{+}}^{q ; \Phi} z(t)=\mathcal{I}_{0^{+}}^{r-q ; \Phi} \mathcal{T}_{z}(t)-\frac{2 b_{2}(\Phi(t)-\Phi(\tau))^{2-q}}{\Gamma(3-q)}
$$

In view of Eq. (3.3) and the boundary condition $\alpha_{1} z(0)+\alpha_{2} z(\xi)=0$, we have

$$
b_{0}=\frac{\alpha_{2}}{\left(\alpha_{1}+\alpha_{2}\right)}\left[\mathcal{I}_{0^{+}}^{r ; \Phi} \mathcal{T}_{z}(\xi)-b_{1}(\Phi(\xi)-\Phi(0))-b_{2}(\Phi(\xi)-\Phi(0))^{2}\right]
$$

Also, from the boundary conditions $\alpha_{1}{ }^{C} \mathcal{D}_{0^{+}}^{p ; \Phi} z(0)+\alpha_{2}{ }^{C} \mathcal{D}_{0^{+}}^{p ; \Phi} z(\xi)=0$ and $\alpha_{1}{ }^{C} \mathcal{D}_{0^{+}}^{q ; \Phi} z(0)+$ $\alpha_{2}{ }^{C} \mathcal{D}_{0^{+}}^{q ; \Phi} z(\xi)=0$ and making use of Eqs. (3.4), (3.5), we obtain

$$
b_{1}=\frac{\Gamma(2-p)}{(\Phi(\xi)-\Phi(0))^{1-p}}\left[\mathcal{I}_{0^{+}}^{r-p ; \Phi} \mathcal{T}_{z}(\xi)-\frac{2 b_{2}(\Phi(\xi)-\Phi(0))^{2-p}}{\Gamma(3-p)}\right]
$$


and

$$
b_{2}=\frac{\Gamma(3-q)}{2(\Phi(\xi)-\Phi(0))^{2-q}} \mathcal{I}_{0^{+}}^{r-q ; \Phi} \mathcal{T}_{z}(\xi)
$$

Substituting $b_{2}$ in Eq. (3.7), we get

$$
b_{1}=\frac{\Gamma(2-p)}{(\Phi(\xi)-\Phi(0))^{1-p}} \mathcal{I}_{0^{+}}^{r-p ; \Phi} \mathcal{T}_{z}(\xi)-\frac{\Gamma(3-q)}{(2-p)(\Phi(\xi)-\Phi(0))^{1-q}} \mathcal{I}_{0^{+}}^{r-q ; \Phi} \mathcal{T}_{z}(\xi)
$$

Inserting $b_{1}, b_{2}$ in Eq. (3.6) gives

$$
\begin{aligned}
b_{0}= & \frac{\alpha_{2}}{\left(\alpha_{1}+\alpha_{2}\right)} \mathcal{I}_{0}^{r ; \Phi} \mathcal{T}_{z}(\xi)-\frac{\alpha_{2} \Gamma(2-p)(\Phi(\xi)-\Phi(0))^{p}}{\left(\alpha_{1}+\alpha_{2}\right)} \mathcal{I}_{0}^{r-p ; \Phi} \mathcal{T}_{z}(\xi) \\
& +\frac{p \alpha_{2} \Gamma(3-q)(\Phi(\xi)-\Phi(0))^{q}}{2\left(\alpha_{1}+\alpha_{2}\right)(2-p)} \mathcal{I}_{0}^{r-q ; \Phi} \mathcal{T}_{z}(\xi) .
\end{aligned}
$$

Make use of Eqs. (3.8), (3.9) and (3.10), in Eq. (3.3), we have

$$
\begin{aligned}
z(t)= & \frac{1}{\Gamma(r)} \int_{0}^{t} \Phi^{\prime}(\tau)(\Phi(t)-\Phi(\tau))^{r-1} \mathcal{T}_{z}(\tau) d \tau \\
& -\frac{\alpha_{2}}{\left(\alpha_{1}+\alpha_{2}\right) \Gamma(r)} \int_{0}^{\xi} \Phi^{\prime}(\tau)(\Phi(\xi)-\Phi(\tau))^{r-1} \mathcal{T}_{z}(\tau) d \tau \\
& +\frac{\Gamma(2-p)\left[\alpha_{2} K_{\Phi}(\xi)-\left(\alpha_{1}+\alpha_{2}\right) K_{\Phi}(t)\right]}{\left(\alpha_{1}+\alpha_{2}\right) \Gamma(r-p)(\Phi(\xi)-\Phi(0))^{1-p}} \int_{0}^{\xi} \Phi^{\prime}(\tau)(\Phi(\xi)-\Phi(\tau))^{r-p-1} \mathcal{T}_{z}(\tau) d \tau \\
& -\left[\frac{\Gamma(3-q)\left[p \alpha_{2}\left(K_{\Phi}(\xi)\right)^{2}-2\left(\alpha_{1}+\alpha_{2}\right) K_{\Phi}(\xi) K_{\Phi}(t)+\left(\alpha_{1}+\alpha_{2}\right)(2-p)\left(K_{\Phi}(t)\right)^{2}\right]}{2\left(\alpha_{1}+\alpha_{2}\right)(2-p) \Gamma(r-q)(\Phi(\xi)-\Phi(0))^{2-q}}\right] \\
& \times \int_{0}^{\xi} \Phi^{\prime}(\tau)(\Phi(\xi)-\Phi(\tau))^{r-q-1} \mathcal{T}_{z}(\tau) d \tau \\
= & \int_{0}^{\xi} \mathcal{H}(t, \tau) \Phi^{\prime}(\tau) \mathcal{T}_{z}(\tau) d \tau .
\end{aligned}
$$

Thus, the result follows. The converse follows directly.

Lemma 3.2 The Green's function $\mathcal{H}(t, \tau)$ defined in (3.2) fulfills the following relationships:

$\left(\mathcal{A}_{1}\right) \mathcal{H}(t, \tau)$ is continuous over $\mathcal{J}$;

$\left(\mathcal{A}_{2}\right) \max _{t \in \mathcal{J}} \int_{0}^{\xi} \mathcal{H}(t, \tau) \Phi^{\prime}(\tau) d \tau \leq \Psi_{1}$, where

$$
\begin{aligned}
\Psi_{1}= & \frac{\alpha_{1}(\Phi(\xi)-\Phi(0))^{r}}{\Gamma(r+1)}+\frac{\alpha_{1} \Gamma(2-p)(\Phi(\xi)-\Phi(0))^{r}}{\left(\alpha_{1}+\alpha_{2}\right) \Gamma(r-p+1)} \\
& +\frac{(p+2) \Gamma(3-q)(\Phi(\xi)-\Phi(0))^{r}}{2(2-p) \Gamma(r-q+1)} .
\end{aligned}
$$

Proof $\left(\mathcal{A}_{1}\right)$ follows trivially. In order to show $\left(\mathcal{A}_{2}\right)$, for any $t \in \mathcal{J}$, we have

$$
\begin{aligned}
& \max _{t \in \mathcal{J}} \int_{0}^{\xi} \mathcal{H}(t, \tau) \Phi^{\prime}(\tau) d \tau \\
& \quad=\max _{t \in \mathcal{J}}\left(\frac{1}{\Gamma(r)} \int_{0}^{t} \Phi^{\prime}(\tau)(\Phi(t)-\Phi(\tau))^{r-1} d \tau\right.
\end{aligned}
$$




$$
\begin{aligned}
& \quad-\frac{\alpha_{2}}{\left(\alpha_{1}+\alpha_{2}\right) \Gamma(r)} \int_{0}^{\xi} \Phi^{\prime}(\tau)(\Phi(\xi)-\Phi(\tau))^{r-1} d \tau \\
& +\frac{\Gamma(2-p)\left[\alpha_{2} K_{\Phi}(\xi)-\left(\alpha_{1}+\alpha_{2}\right) K_{\Phi}(t)\right]}{\left(\alpha_{1}+\alpha_{2}\right) \Gamma(r-p)(\Phi(\xi)-\Phi(0))^{1-p}} \int_{0}^{\xi} \Phi^{\prime}(\tau)(\Phi(\xi)-\Phi(\tau))^{r-p-1} d \tau \\
& -\frac{\Gamma(3-q)\left[p \alpha_{2}\left(K_{\Phi}(\xi)\right)^{2}-2\left(\alpha_{1}+\alpha_{2}\right) K_{\Phi}(\xi) K_{\Phi}(t)+\left(\alpha_{1}+\alpha_{2}\right)(2-p)\left(K_{\Phi}(t)\right)^{2}\right]}{2\left(\alpha_{1}+\alpha_{2}\right)(2-p) \Gamma(r-q)(\Phi(\xi)-\Phi(0))^{2-q}} \\
& \left.\quad \times \int_{0}^{T} \Phi^{\prime}(\tau)(\Phi(\xi)-\Phi(\tau))^{r-q-1} d \tau\right) \\
& \leq \max _{t \in \mathcal{J}}\left(\frac{\alpha_{1}(\Phi(t)-\Phi(0))^{r}}{\Gamma(r+1)}+\frac{\Gamma(2-p)\left[\alpha_{2}(\Phi(\xi)-\Phi(0))-\left(\alpha_{1}+\alpha_{2}\right)(\Phi(t)-\Phi(0))\right]}{\left(\alpha_{1}+\alpha_{2}\right) \Gamma(r-p+1)(\Phi(\xi)-\Phi(0))^{1-r}}\right. \\
& \left.\quad-\frac{\Gamma(3-q)\left[p \alpha_{2}\left(K_{\Phi}(\xi)\right)^{2}-2\left(\alpha_{1}+\alpha_{2}\right) K_{\Phi}(\xi) K_{\Phi}(t)+\left(\alpha_{1}+\alpha_{2}\right)(2-p)\left(K_{\Phi}(t)\right)^{2}\right]}{2\left(\alpha_{1}+\alpha_{2}\right)(2-p) \Gamma(r-q+1)(\Phi(T)-\Phi(0))^{2-r}}\right) \\
& \leq \frac{\alpha_{1}(\Phi(\xi)-\Phi(0))^{r}}{\Gamma(r+1)}+\frac{\alpha_{1} \Gamma(2-p)(\Phi(\xi)-\Phi(0))^{r}}{\left(\alpha_{1}+\alpha_{2}\right) \Gamma(r-p+1)}+\frac{(p+2) \Gamma(3-q)(\Phi(\xi)-\Phi(0))^{r}}{2(2-p) \Gamma(r-q+1)} \\
& \leq \Psi_{1} .
\end{aligned}
$$

\subsection{Existence result}

In this subsection, we use Theorem 2.7 to establish the existence of at least one solution to the problem (1.4) on $\mathcal{J}$.

\section{Theorem 3.3 Suppose that}

$\left(\mathcal{A}_{3}\right)$ There exist $\lambda_{0}, \lambda_{1}, \lambda_{2} \in \mathcal{C}([\mathcal{J}, \mathbb{R}])$ with $\lambda_{0}^{*}=\sup _{t \in \mathcal{J}}\left|\lambda_{0}(t)\right|, \lambda_{1}^{*}=\sup _{t \in \mathcal{J}}\left|\lambda_{1}(t)\right|, \lambda_{2}^{*}=$ $\sup _{t \in \mathcal{J}}\left|\lambda_{2}(t)\right|<1$, such that

$$
\left|f\left(t, u_{1}, u_{2}, u_{3}\right)\right| \leq \lambda_{0}(t)+\lambda_{1}(t)\left[\left|u_{1}\right|+\left|u_{2}\right|\right]+\lambda_{2}(t)\left|u_{3}\right|
$$

for any $u_{1}, u_{2}, u_{3} \in \mathbb{R}$ and $t \in \mathcal{J}$. Then the proposed problem (1.4) has at least one solution on $\mathcal{J}$.

Proof We viewed problem (1.4) as a fixed point problem by taking into consideration the operator $\mathcal{N}: \mathbb{E} \rightarrow \mathbb{E}$ defined by

$$
(\mathcal{N} z)(t)=\int_{0}^{\xi} \mathcal{H}(t, \tau) \Phi^{\prime}(\tau) \mathcal{T}_{z} d \tau \quad \text { for each } t \in \mathcal{J}
$$

Obviously, it is not difficult to show that the operator $\mathcal{N}$ is well defined and the fixed point of $\mathcal{N}$ is the solution to the problem (1.4). Thus, using Theorem 2.7, we show that the operator $\mathcal{N}$ has at least one fixed point.

The proof shall be given in the following manner.

Claim $1 \mathcal{N}$ is continuous. 
Suppose that $z_{n} \rightarrow z$ is a convergent sequence in $\mathbb{E}$. Thus, for $t \in \mathcal{J}$ and using assumption $\left(\mathcal{A}_{2}\right)$ yields

$$
\begin{aligned}
\left|\left(\left(\mathcal{N} z_{n}\right)(t)-(\mathcal{N} z)(t)\right)\right| & =\left|\int_{0}^{\xi} \mathcal{H}(t, \tau) \Phi^{\prime}(\tau)\left(\mathcal{T}_{z_{n}}(\tau)-\mathcal{T}_{z}(\tau)\right) d \tau\right| \\
& \leq \int_{0}^{\xi}|\mathcal{H}(t, \tau)| \Phi^{\prime}(\tau)\left|\mathcal{T}_{z_{n}}(\tau)-\mathcal{T}_{z}(\tau)\right| d \tau \\
& \leq \Psi_{1}\left|\mathcal{T}_{z_{n}}(\tau)-\mathcal{T}_{z}(\tau)\right| .
\end{aligned}
$$

Since $\mathcal{T}_{z}$ is continuous, we get

$$
\left\|\mathcal{N}_{z_{n}}-\mathcal{N}_{z}\right\|_{\mathbb{E}} \rightarrow 0, \quad \text { as } n \rightarrow \infty
$$

Claim $2 \mathcal{N}$ maps bounded sets into bounded sets.

Let $\gamma>0$, and construct a closed convex set $z \in \mathcal{B}_{\gamma}=\left\{z \in \mathbb{E}:\|z\|_{\mathbb{E}} \leq \gamma\right\}$. Then we show that there exists $\eta>0$ such that $\|\mathcal{N}(z)\|_{\mathbb{E}} \leq \eta$.

Indeed, for any $z \in \mathcal{B}_{\gamma}$,

$$
\begin{aligned}
|\mathcal{N}(z)(t)| & =\left|\int_{0}^{\xi} \mathcal{H}(t, \tau) \Phi^{\prime}(\tau) \mathcal{T}_{z}(\tau) d \tau\right| \\
& \leq \int_{0}^{\xi}|\mathcal{H}(t, \tau)| \Phi^{\prime}(\tau)\left|\mathcal{T}_{z}(\tau)\right| d \tau .
\end{aligned}
$$

It follows from assumption $\left(\mathcal{A}_{3}\right)$ that

$$
\begin{aligned}
\left|\mathcal{T}_{z}(t)\right| & =\left|f\left(t, z(t), z(\sigma t), \mathcal{T}_{z}(t)\right)\right| \\
& \leq \lambda_{0}(t)+\lambda_{1}(t)(|z(t)|+|z(\sigma t)|)+\lambda_{2}(t)\left|\mathcal{T}_{z}(t)\right| \\
& \leq \frac{\lambda_{0}^{*}+2 \lambda_{1}^{*}\|z\|_{\mathbb{E}}}{1-\lambda_{2}^{*}} \\
& \leq \frac{\lambda_{0}^{*}+2 \lambda_{1}^{*} \gamma}{1-\lambda_{2}^{*}} .
\end{aligned}
$$

In view of Eqs. (3.15) and (3.16), we get

$$
|\mathcal{N}(z)(t)| \leq\left(\frac{\lambda_{0}^{*}+2 \lambda_{1}^{*} \gamma}{1-\lambda_{2}^{*}}\right)\left(\int_{0}^{\xi}|\mathcal{H}(t, \tau)| \Phi^{\prime}(\tau) d \tau\right) .
$$

Using property $\left(\mathcal{A}_{2}\right)$ and inequality (3.17) yields

$$
\begin{aligned}
\|\mathcal{N}(z)\|_{\mathbb{E}} & \leq\left(\frac{\lambda_{0}^{*}+2 \lambda_{1}^{*} \gamma}{1-\lambda_{2}^{*}}\right) \Psi_{1} \\
& =\eta<\infty .
\end{aligned}
$$

Claim $3 \mathcal{N}$ maps a bounded set into an equicontinuous set. 
Suppose $t_{1}, t_{2} \in \mathcal{J}$ such that $t_{2} \geq t_{1}$ and $\mathcal{B}_{\gamma}$ as defined in Claim 2, above. So, for any $z \in \mathcal{B}_{\gamma}$, we obtain

$$
\begin{aligned}
&\left|(\mathcal{N} z)\left(t_{2}\right)-(\mathcal{N} z)\left(t_{1}\right)\right| \\
& \leq \frac{1}{\Gamma(r)} \int_{0}^{t_{1}} \Phi^{\prime}(\tau)\left[\left(\Phi\left(t_{1}\right)-\Phi(\tau)\right)^{r-1}-\left(\Phi\left(t_{2}\right)-\Phi(\tau)\right)^{r-1}\right]\left|\mathcal{T}_{z}(\tau)\right| d \tau \\
&+\frac{1}{\Gamma(r)} \int_{t_{1}}^{t_{2}} \Phi^{\prime}(\tau)\left(\Phi\left(t_{2}\right)-\Phi(\tau)\right)^{r-1}\left|\mathcal{T}_{z}(\tau)\right| d \tau \\
&+\frac{\Gamma(2-q)\left(\Phi\left(t_{2}\right)-\Phi\left(t_{1}\right)\right)}{\Gamma(r-p)(\Phi(\xi)-\Phi(0))^{1-p}} \int_{0}^{\xi} \Phi^{\prime}(\tau)(\Phi(\xi)-\Phi(\tau))^{r-p-1}\left|\mathcal{T}_{z}(\tau)\right| d \tau \\
&+\frac{\Gamma(3-q)\left(\Phi\left(t_{2}\right)-\Phi\left(t_{1}\right)\right)}{(2-p) \Gamma(r-q)(\Phi(\xi)-\Phi(0))^{1-q}} \int_{0}^{\xi} \Phi^{\prime}(\tau)(\Phi(\xi)-\Phi(\tau))^{r-q-1}\left|\mathcal{T}_{z}(\tau)\right| d \tau \\
&+\frac{\Gamma(3-q)\left(\Phi\left(t_{2}\right)-\Phi\left(t_{1}\right)\right)^{2}}{2 \Gamma(r-q)(\Phi(\xi)-\Phi(0))^{2-q}} \int_{0}^{\xi} \Phi^{\prime}(\tau)(\Phi(\xi)-\Phi(\tau))^{r-q-1}\left|\mathcal{T}_{z}(\tau)\right| d \tau \\
& \leq \frac{2\left(\Phi\left(t_{2}\right)-\Phi\left(t_{1}\right)\right)^{r}-\left[\left(\Phi\left(t_{2}\right)-\Phi(0)\right)^{r}-\left(\Phi\left(t_{1}\right)-\Phi(0)\right)^{r}\right]}{\Gamma(r+1)} \\
&+\frac{\Gamma(2-q)\left(\Phi\left(t_{2}\right)-\Phi\left(t_{1}\right)\right)}{\Gamma(r-p+1)}(\Phi(\xi)-\Phi(0))^{r-1} \\
&+\frac{\Gamma(3-q)\left(\Phi\left(t_{2}\right)-\Phi\left(t_{1}\right)\right)}{(2-p) \Gamma(r-q+1)}(\Phi(\xi)-\Phi(0))^{r-1} \\
&\left.+\frac{\Gamma(3-q)\left(\Phi\left(t_{2}\right)-\Phi\left(t_{1}\right)\right)^{2}}{2 \Gamma(r-q+1)}(\Phi(\xi)-\Phi(0))^{r-1}\right]\left(\frac{\lambda_{0}^{*}+2 \lambda_{1}^{*} \gamma}{1-\lambda_{2}^{*}}\right) \\
& \rightarrow 0, \quad \text { as } t_{1} \rightarrow t_{2} .
\end{aligned}
$$

Hence, as a consequence of the Arzelá-Ascoli theorem and Claims 1-3, the operator $\mathcal{N}$ is completely continuous.

Claim $4 \mathcal{N}$ is a priori bounded.

Now, consider the set

$$
\vartheta=\{z \in \mathbb{E}: z=\varepsilon(\mathcal{N} z), 0<\varepsilon<1\}
$$

Then it is enough to show that $\vartheta$ is bounded.

Indeed, for any $z \in \vartheta, z=\varepsilon(\mathcal{N} z)$ where $0<\varepsilon<1$, we have

$$
z(t)=\varepsilon\left[\int_{0}^{\xi} \mathcal{H}(t, \tau) \Phi^{\prime}(\tau) \mathcal{T}_{z}(\tau) d \tau\right]
$$

It follows from $\left(\mathcal{A}_{2}\right)$ and inequality (3.16) that, for each $t \in \mathcal{J}$,

$$
\begin{aligned}
|z(t)| & =|\varepsilon(\mathcal{N} z)(t)| \\
& \leq \max _{t \in \mathcal{J}} \int_{0}^{\xi}|\mathcal{H}(t, \tau)| \Phi^{\prime}(\tau)\left|\mathcal{T}_{z}(\tau)\right| d \tau \\
& =\eta<+\infty .
\end{aligned}
$$


Thus, the set $\vartheta$ is bounded. As a result of Theorem 2.7, the operator $\mathcal{N}$ has at least one fixed point. Thus, we conclude that problem (1.4) has at least one solution on $\mathcal{J}$.

\subsection{Uniqueness result}

This subsection gives a detail proof of the uniqueness of solution of problem (1.4) by applying the concept of Banach contraction principle.

Theorem 3.4 Suppose that we have the hypotheses:

$\left(\mathcal{A}_{4}\right)$ There exist constants $\mathcal{K}_{1}, \mathcal{K}_{2}>0$ such that

$$
\begin{aligned}
\left|f\left(t, u_{1}, u_{2}, u_{3}\right)-f\left(t, \bar{u}_{1}, \bar{u}_{2}, \bar{u}_{3}\right)\right| \leq & \mathcal{K}_{1}\left(\left|u_{1}-\bar{u}_{1}\right|+\left|u_{2}-\bar{u}_{2}\right|\right) \\
& +\mathcal{K}_{2}\left|u_{3}-\bar{u}_{3}\right|
\end{aligned}
$$

for any $u_{1}, u_{2}, u_{3}, \bar{u}_{1}, \bar{u}_{2}, \bar{u}_{3} \in \mathbb{R}$ and $t \in \mathcal{J}$.

$\left(\mathcal{A}_{5}\right)$ We assume

$$
\left(\frac{2 \mathcal{K}_{1}}{1-\mathcal{K}_{2}}\right) \Psi_{1}<1
$$

where $\Psi_{1}$ is given by (3.12). If there exists a solution of the proposed problem (1.4), it is unique.

Proof Consider the operator $\mathcal{N}$ as defined in (3.13). Let $z_{1}, z_{2} \in \mathbb{E}$ and $t \in \mathcal{J}$, then we obtain

$$
\begin{aligned}
\left|\left(\mathcal{N} z_{1}\right)(t)-\left(\mathcal{N} z_{2}\right)(t)\right| & =\left|\int_{0}^{\xi} \mathcal{H}(t, \tau) \Phi^{\prime}(\tau)\left(\mathcal{T}_{z_{1}}(\tau)-\mathcal{T}_{z_{2}}(\tau)\right) d \tau\right| \\
& \leq \int_{0}^{\xi}|\mathcal{H}(t, \tau)| \Phi^{\prime}(\tau)\left|\mathcal{T}_{z_{1}}(\tau)-\mathcal{T}_{z_{2}}(\tau)\right| d \tau
\end{aligned}
$$

and

$$
\begin{aligned}
\left|\mathcal{T}_{z_{1}}(t)-\mathcal{T}_{z_{2}}(t)\right| & =\left|f\left(t, z_{1}(t), z_{1}(\sigma t), \mathcal{T}_{z_{1}}(t)\right)-f\left(t, z_{2}(t), z_{2}(\sigma t), \mathcal{T}_{z_{2}}(t)\right)\right| \\
& \leq \mathcal{K}_{1}\left(\left|z_{1}(t)-z_{2}(t)\right|+\left|z_{1}(\sigma t)-z_{2}(\sigma t)\right|\right)+\mathcal{K}_{2}\left|\mathcal{T}_{z_{1}}(t)-\mathcal{T}_{z_{2}}(t)\right| \\
& \leq\left(\frac{2 \mathcal{K}_{1}}{1-\mathcal{K}_{2}}\right)\left|z_{1}(t)-z_{2}(t)\right|
\end{aligned}
$$

By substituting inequality (3.22) in (3.21), we get

$$
\begin{aligned}
\left|\left(\mathcal{N} z_{1}\right)(t)-\left(\mathcal{N} z_{2}\right)(t)\right| & \leq\left(\frac{2 \mathcal{K}_{1}}{1-\mathcal{K}_{1}}\right)\left(\int_{0}^{\xi}|\mathcal{H}(t, \tau)| \Phi^{\prime}(\tau) d \tau\right)\left|z_{1}(t)-z_{2}(t)\right| \\
& \leq\left(\frac{2 \mathcal{K}_{1}}{1-\mathcal{K}_{2}}\right)\left(\max _{t \in \mathcal{J}} \int_{0}^{\xi}|\mathcal{H}(t, \tau)| \Phi^{\prime}(\tau) d \tau\right)\left|z_{1}(t)-z_{2}(t)\right| \\
& \leq\left(\frac{2 \mathcal{K}_{1}}{1-\mathcal{K}_{2}}\right) \Psi_{1}\left|z_{1}(t)-z_{2}(t)\right| .
\end{aligned}
$$


Thus,

$$
\left\|\left(\mathcal{N} z_{1}\right)-\left(\mathcal{N} z_{2}\right)\right\|_{\mathbb{E}} \leq\left(\frac{2 \mathcal{K}_{1}}{1-\mathcal{K}_{2}}\right) \Psi_{1}\left\|z_{1}-z_{2}\right\|_{\mathbb{E}}
$$

In view of $\left(\mathcal{A}_{5}\right)$, this that $\mathcal{N}$ is a contraction and hence has a unique fixed point. Therefore, as a consequences of Theorem 2.8, the proposed problem (1.4) has a unique solution on $\mathcal{J}$.

The $\Phi$-Caputo fractional derivative as described Definition 2.4 comprises, as special cases, certain specific forms (definitions) of fractional derivatives. Thus, the nonlinear implicit fractional pantograph differential equation (1.4) that we have just proposed also incorporates, as special cases, equivalent nonlinear Cauchy problems for certain forms of fractional derivatives (see Remark 1.1). Besides, the results we have just established on the existence and uniqueness of its solution also apply to special cases. These classes of derivatives are obtained based on the choices of the arbitrary function $\Phi(\cdot)$ and the parameters $\alpha_{1}, \alpha_{2}$.

Case 1: [8] Set $\alpha_{1}=\alpha_{2}=1$ and $\Phi(t)=t$. Then the Green's function defined in (3.2) takes the form

$$
\mathcal{H}^{*}(t, \tau)=\left\{\begin{array}{cl}
\frac{2(t-\tau)^{r-1}-(\xi-\tau)^{r-1}}{2 \Gamma(r)}+\frac{\Gamma(2-p)(\xi-2 t)}{2 \Gamma(r-p) \xi^{1-p}}(\xi-\tau)^{r-p-1} & \\
-\frac{\Gamma(3-q)\left[p \xi^{2}-4 \xi t+2(2-p) t^{2}\right]}{4(2-p) \Gamma(r-q) \xi^{1-p}}(\xi-\tau)^{r-q-1}, & 0 \leq \tau \leq t \leq \xi ; \\
\frac{(\xi-\tau)^{r-1}}{2 \Gamma(r)}+\frac{\Gamma(2-p)(\xi-2 t)}{2 \Gamma(r-p) \xi^{1-p}}(\xi-\tau)^{r-p-1} & \\
-\frac{\Gamma(3-q)\left[p \xi^{2}-4 \xi t+2(2-p) t^{2}\right]}{4(2-p) \Gamma(r-q) \xi^{1-p}}(\xi-\tau)^{r-q-1}, & 0 \leq t \leq \tau \leq \xi,
\end{array}\right.
$$

and

$$
\Psi_{1}^{*}=\frac{\xi^{r}}{\Gamma(r+1)}+\frac{\Gamma(2-p) \xi^{r}}{2 \Gamma(r-p+1)}+\frac{(p+2) \Gamma(3-q) \xi^{r}}{2(2-p) \Gamma(r-q+1)} .
$$

Corollary 3.5 Suppose that the assumption $\left(\mathcal{A}_{3}\right)$ holds. Then the proposed problem (1.4) has at least one solution on $\mathcal{J}$.

Corollary 3.6 Assume that hypothesis $\left(\mathcal{A}_{4}\right)$ holds. If

$$
\left(\frac{2 \mathcal{K}_{1}}{1-\mathcal{K}_{2}}\right) \Psi_{1}^{*}<1
$$

where $\Psi_{1}^{*}$ is defined by (3.26). If there exists a solution of the proposed problem (1.4), it is unique on $\mathcal{J}$. 
Case 2: Let $\alpha_{1} \neq \alpha_{2}>1$ and $\Phi(t)=t$, then the Green's function defined in (3.2) reduces to

$$
\mathcal{H}^{* *}(t, \tau)=\left\{\begin{array}{c}
\frac{\left(\alpha_{1}+\alpha_{2}\right)(t-\tau)^{r-1}-\alpha_{2}(\xi-\tau)^{r-1}}{\left(\alpha_{1}+\alpha_{2}\right) \Gamma(r)}+\frac{\Gamma(2-p)\left[\alpha_{2} \xi-\left(\alpha_{1}+\alpha_{2}\right) t\right]}{\left(\alpha_{1}+\alpha_{2}\right) \Gamma(r-p) \xi^{1-p}}(\xi-\tau)^{r-p-1} \\
-\frac{\Gamma(3-q)\left[p \alpha_{2} \xi^{2}-2\left(\alpha_{1}+\alpha_{2}\right) \xi t+\left(\alpha_{1}+\alpha_{2}\right)(2-p) t^{2}\right]}{2\left(\alpha_{1}+\alpha_{2}\right)(2-p) \Gamma(r-q) \xi^{1-p}}(\xi-\tau)^{r-q-1}, \\
0 \leq \tau \leq t \leq \xi \\
\frac{\alpha_{2}(\xi-\tau)^{r-1}}{\left(\alpha_{1}+\alpha_{2}\right) \Gamma(r)}+\frac{\Gamma(2-p)\left[\alpha_{2} \xi-\left(\alpha_{1}+\alpha_{2}\right) t\right]}{\left(\alpha_{1}+\alpha_{2}\right) \Gamma(r-p) \xi^{1-p}}(\xi-\tau)^{r-p-1} \\
-\frac{\Gamma(3-q)\left[p \alpha_{2} \xi^{2}-2\left(\alpha_{1}+\alpha_{2}\right) \xi t+\left(\alpha_{1}+\alpha_{2}\right)(2-p) t^{2}\right]}{2\left(\alpha_{1}+\alpha_{2}\right)(2-p) \Gamma(r-q) \xi^{1-p}}(\xi-\tau)^{r-q-1}, \\
0 \leq t \leq \tau \leq \xi,
\end{array}\right.
$$

and

$$
\Psi_{1}^{* *}=\frac{\alpha_{1} \xi^{r}}{\Gamma(r+1)}+\frac{\alpha_{1} \Gamma(2-p) \xi^{r}}{\left(\alpha_{1}+\alpha_{2}\right) \Gamma(r-p+1)}+\frac{(p+2) \Gamma(3-q) \xi^{r}}{2(2-p) \Gamma(r-q+1)}
$$

Corollary 3.7 Suppose that the assumption $\left(\mathcal{A}_{3}\right)$ holds. Then the proposed problem (1.4) has at least one solution on $\mathcal{J}$.

Corollary 3.8 Assume that hypothesis $\left(\mathcal{A}_{4}\right)$ holds. If

$$
\left(\frac{2 \mathcal{K}_{1}}{1-\mathcal{K}_{2}}\right) \Psi_{1}^{* *}<1
$$

where $\Psi_{1}^{* *}$ is defined by (3.28), and if there exists a solution of the proposed problem (1.4), it is unique on $\mathcal{J}$.

Lemma 3.9 Let $1<r \leq 2$ and $f: \mathcal{J} \times \mathbb{R}^{3}$ be a continuous function for any $z \in \mathcal{A C}_{\Phi}^{2}([\mathcal{J}, \mathbb{R}])$. A function $z \in \mathcal{A C}_{\Phi}^{2}([\mathcal{J}, \mathbb{R}])$ is a solution of problem (1.5) if and only if $z$ satisfies the following integral equation:

$$
z(t)=\int_{0}^{\xi} \mathcal{W}(t, \tau) \Phi^{\prime}(\tau) f\left(t, z(\tau), z(\sigma \tau), \mathcal{T}_{z}(\tau)\right) d \tau
$$

where $\mathcal{W}(t, \tau)$ is a Green's function of the form

$$
\mathcal{W}(t, \tau)=\left\{\begin{array}{l}
\frac{\left(\alpha_{1}+\alpha_{2}\right)(\Phi(t)-\Phi(\tau))^{r-1}-\alpha_{2}(\Phi(\xi)-\Phi(\tau))^{r-1}}{\left(\alpha_{1}+\alpha_{2}\right) \Gamma(r)} \\
\quad+\frac{\left[\alpha_{2}^{2}(\Phi(\xi)-\Phi(0))-\alpha_{2}\left(\alpha_{1}+\alpha_{2}\right)(\Phi(t)-\Phi(0))\right]}{\left(\alpha_{1}+\alpha_{2}\right)^{2} \Gamma(r-1)}(\Phi(\xi)-\Phi(\tau))^{r-2}, \\
0 \leq \tau \leq t \leq \xi ; \\
-\frac{\alpha_{2}(\Phi(\xi)-\Phi(\tau))^{r-1}}{\left(\alpha_{1}+\alpha_{2}\right) \Gamma(r)}+\frac{\left[\alpha_{2}^{2}(\Phi(\xi)-\Phi(0))-\alpha_{2}\left(\alpha_{1}+\alpha_{2}\right)(\Phi(t)-\Phi(0))\right]}{\left(\alpha_{1}+\alpha_{2}\right)^{2} \Gamma(r-1)} \\
\quad \times(\Phi(\xi)-\Phi(\tau))^{r-2}, \quad 0 \leq t \leq \tau \leq \xi .
\end{array}\right.
$$

Proof The proof is a partial case of Lemma 3.1, so we omit the proof.

Lemma 3.10 The Green's function $\mathcal{W}(t, \tau)$ defined in (3.30) adhere to the following conditions:

$\left(\mathcal{A}_{6}\right) \mathcal{W}(t, \tau)$ is continuous over $\mathcal{J}$; 
$\left(\mathcal{A}_{7}\right) \max _{t \in \mathcal{J}} \int_{0}^{\xi} \mathcal{W}(t, \tau) \Phi^{\prime}(\tau) d \tau \leq \Psi_{2}$, where

$$
\Psi_{2}=\frac{\left[\left(\alpha_{1}+2 \alpha_{2}\right)\left(\alpha_{1}+\alpha_{2}\right)+\alpha_{2}^{2} r\right]}{\left(\alpha_{1}+\alpha_{2}\right)^{2} \Gamma(r+1)}(\Phi(\xi)-\Phi(0))^{r} .
$$

Proof Obviously, for any $t \in \mathcal{J}$, the Green's function $\mathcal{W}(t, \tau)$ is continuous. Thus, $\left(\mathcal{A}_{6}\right)$ follows.

In order to prove $\left(\mathcal{A}_{7}\right)$, for any $t \in \mathcal{J}$, we write

$$
\begin{aligned}
& \max _{t \in \mathcal{J}} \int_{0}^{\xi} \mathcal{W}(t, \tau) \Phi^{\prime}(\tau) d \tau \\
&=\max _{t \in \mathcal{J}}\left[\frac{1}{\Gamma(r)} \int_{0}^{\xi} \Phi^{\prime}(\tau)(\Phi(\xi)-\Phi(\tau))^{r-1} d \tau\right. \\
& \quad-\frac{\alpha_{2}}{\left(\alpha_{1}+\alpha_{2}\right) \Gamma(r)} \int_{0}^{\xi} \Phi^{\prime}(\tau)(\Phi(t)-\Phi(\tau))^{r-1} d \tau \\
&\left.\quad+\frac{\left[\alpha_{2}^{2}(\Phi(\xi)-\Phi(0))-\alpha_{2}\left(\alpha_{1}+\alpha_{2}\right)(\Phi(t)-\Phi(0))\right]}{\left(\alpha_{1}+\alpha_{2}\right)^{2} \Gamma(r-1)} \int_{0}^{\xi} \Phi^{\prime}(\tau)(\Phi(\xi)-\Phi(\tau))^{r-2} d \tau\right] \\
& \leq \max _{t \in \mathcal{J}}\left[\frac{\left(\alpha_{1}+2 \alpha_{2}\right)\left(\alpha_{1}+\alpha_{2}\right)(\Phi(\xi)-\Phi(\tau))^{r}}{\left(\alpha_{1}+\alpha_{2}\right) \Gamma(r+1)}\right. \\
&\left.\quad+\frac{\left[\alpha_{2}^{2}(\Phi(\xi)-\Phi(0))-\alpha_{2}\left(\alpha_{1}+\alpha_{2}\right)(\Phi(t)-\Phi(0))\right]}{\left(\alpha_{1}+\alpha_{2}\right)^{2} \Gamma(r)}(\Phi(\xi)-\Phi(\tau))^{r-1}\right] \\
& \leq \frac{\left(\alpha_{1}+2 \alpha_{2}\right)\left(\alpha_{1}+\alpha_{2}\right)(\Phi(\xi)-\Phi(\tau))^{r}}{\left(\alpha_{1}+\alpha_{2}\right) \Gamma(r+1)}+\frac{\alpha_{2}^{2}(\Phi(\xi)-\Phi(0))^{r}}{\left(\alpha_{1}+\alpha_{2}\right)^{2} \Gamma(r)} \\
&= \frac{\left[\left(\alpha_{1}+2 \alpha_{2}\right)\left(\alpha_{1}+\alpha_{2}\right)+\alpha_{2}^{2} r\right]}{\left(\alpha_{1}+\alpha_{2}\right)^{2} \Gamma(r+1)}(\Phi(\xi)-\Phi(0))^{r} \\
&= \Psi_{2} .
\end{aligned}
$$

Hence, we have the desired result.

Theorem 3.11 Suppose that the assumption $\left(\mathcal{A}_{3}\right)$ holds. Then the proposed problem (1.5) has at least one solution.

Proof Repeating the analysis in Theorem 3.3, the proof follows.

Theorem 3.12 Assume that hypothesis $\left(\mathcal{A}_{4}\right)$ holds and

$$
\left(\frac{2 \mathcal{K}_{1}}{1-\mathcal{K}_{2}}\right) \Psi_{2}<1
$$

where $\Psi_{2}$ is defined by (3.31), then, if there exists a solution of (1.5), it is unique on $\mathcal{J}$.

Proof Repeating the analysis in Theorem 3.4, the proof follows.

Now we present some special cases of the proposed problem (1.5) based on the choice of the arbitrary function $\Phi$ and parameters $\alpha_{1}, \alpha_{2}$. 
Case 3: [2, 3, 14] Let $\alpha_{1}=\alpha_{2}=1$ and $\Phi(t)=t$. Then the Green's function defined in (3.30) reduces to

$$
\mathcal{W}^{*}(t, \tau)=\left\{\begin{array}{l}
\frac{2(t-\tau)^{r-1}-(\xi-\tau)^{r-1}}{2 \Gamma(r)}+\frac{(\xi-2 t)}{4 \Gamma(r-1)}(\xi-\tau)^{r-2}, \quad 0 \leq \tau \leq t \leq \xi \\
-\frac{(\xi-\tau)^{r-1}}{2 \Gamma(r)}+\frac{(\xi-2 t)}{4 \Gamma(r-1)}(\xi-\tau)^{r-2}, \quad 0 \leq t \leq \tau \leq \xi
\end{array}\right.
$$

and

$$
\Psi_{2}^{*}=\left(\frac{3}{2 \Gamma(r+1)}+\frac{1}{2 \Gamma(r)}\right) \xi^{r}
$$

Corollary 3.13 Suppose that the assumption $\left(\mathcal{A}_{3}\right)$ holds. Then the proposed problem (1.5) has at least one solution on $\mathcal{J}$.

Corollary 3.14 Assume that hypothesis $\left(\mathcal{A}_{4}\right)$ holds. If

$$
\left(\frac{2 \mathcal{K}_{1}}{1-\mathcal{K}_{2}}\right) \Psi_{2}^{*}<1
$$

where $\Psi_{2}^{*}$ is defined by (3.34). If there exists a solution of (1.5), it is unique on $\mathcal{J}$.

Moreover, if $r=2$ the Green's function defined in (3.30) reduces to [33]

$$
\mathcal{W}^{* *}(t, \tau)= \begin{cases}\frac{1}{4}(-\xi-2 t+2 \tau), & \text { if } 0 \leq \tau \leq t \leq \xi \\ \frac{1}{4}(-\xi+2 t-2 \tau), & \text { if } 0 \leq t \leq \tau \leq \xi\end{cases}
$$

Case 4: Suppose $\alpha_{1} \neq \alpha_{2}>1$ and $\Phi(t)=t$, then the Green's function defined in (3.30) takes the form

$$
\mathcal{W}^{* * *}(t, \tau)=\left\{\begin{array}{l}
\frac{\left(\alpha_{1}+\alpha_{2}\right)(t-\tau)^{r-1}-\alpha_{2}(\xi-\tau)^{r-1}}{\left(\alpha_{1}+\alpha_{2}\right) \Gamma(r)}+\frac{\left[\alpha_{2}^{2} \xi-\alpha_{2}\left(\alpha_{1}+\alpha_{2}\right) t\right]}{\left(\alpha_{1}+\alpha_{2}\right)^{2} \Gamma(r-1)}(\xi-\tau)^{r-2}, \\
0 \leq \tau \leq t \leq \xi ; \\
-\frac{\alpha_{2}(\xi-\tau)^{r-1}}{\left(\alpha_{1}+\alpha_{2}\right) \Gamma(r)}+\frac{\left[\alpha_{2}^{2} \xi-\alpha_{2}\left(\alpha_{1}+\alpha_{2}\right) t\right]}{\left(\alpha_{1}+\alpha_{2}\right)^{2} \Gamma(r-1)}(\xi-\tau)^{r-2}, \quad 0 \leq t \leq \tau \leq \xi,
\end{array}\right.
$$

and

$$
\Psi_{2}^{* *}=\frac{\left[\left(\alpha_{1}+2 \alpha_{2}\right)\left(\alpha_{1}+\alpha_{2}\right)+\alpha_{2}^{2} r\right]}{\left(\alpha_{1}+\alpha_{2}\right)^{2} \Gamma(r+1)} \xi^{r}
$$

Corollary 3.15 Suppose that assumption $\left(\mathcal{A}_{3}\right)$ holds. Then the proposed problem $(1.5)$ has at least one solution on $\mathcal{J}$.

Corollary 3.16 Assume that hypothesis $\left(\mathcal{A}_{4}\right)$ holds. If

$$
\left(\frac{2 \mathcal{K}_{1}}{1-\mathcal{K}_{2}}\right) \Psi_{2}^{* *}<1
$$

where $\Psi_{2}^{* *}$ is defined by (3.36), if there exists a solution of (1.5), it is unique on $\mathcal{J}$. 


\subsection{Examples}

Example 3.17 Consider the IFPBVPs of the form

$$
\left\{\begin{array}{l}
{ }^{C} \mathcal{D}_{0^{+}}^{\frac{7}{3}, \Phi} z(t)=\frac{1}{(t+2)^{4}\left[1+|z(t)|+\left|z\left(\frac{2}{7} t\right)\right|+\left|{ }^{C} \mathcal{D}_{0^{+}}^{\frac{7}{3}, \Phi} z(t)\right|\right]}, \quad t \in \mathcal{J}=[0,1] \\
2 z(0)+4 z(1)=0, \quad 2^{C} \mathcal{D}_{0^{+}}^{\frac{1}{3} ; \Phi} z(0)+4^{C} \mathcal{D}_{0^{+}}^{\frac{1}{3} ; \Phi} z(1)=0 \\
2^{C} \mathcal{D}_{0^{+}}^{\frac{5}{3} ; \Phi} z(0)+4^{C} \mathcal{D}_{0^{+}}^{\frac{5}{3} ; \Phi} z(1)=0 .
\end{array}\right.
$$

Comparing (1.4) with (3.37) yields $r=\frac{7}{3}, p=\frac{1}{3}, q=\frac{5}{3}, \xi=1$, and $\sigma=\frac{2}{7}$. Also from the boundary conditions we can see that $\alpha_{1}=2$ and $\alpha_{2}=4$. Here, the function $f: \mathcal{J} \times \mathbb{R}^{3} \rightarrow \mathbb{R}$ defined by

$$
f\left(t, u_{1}, u_{2}, u_{3}\right)=\frac{1}{(t+2)^{4}\left(1+\left|u_{1}\right|+\left|u_{2}\right|+\left|u_{3}\right|\right)}, \quad t \in \mathcal{J}, u_{1}, u_{2}, u_{3} \in \mathbb{R}_{+},
$$

is continuous and

$$
\left|f\left(t, u_{1}, u_{2}, u_{3}\right)\right| \leq \frac{1}{(t+2)^{4}}\left(1+\left|u_{1}\right|+\left|u_{2}\right|+\left|u_{3}\right|\right), \quad t \in \mathcal{J}
$$

so condition $\left(\mathcal{A}_{3}\right)$ is satisfied with $\lambda_{0}(t)=\lambda_{1}(t)=\lambda_{2}(t)=\frac{1}{(t+2)^{4}}$ and $\lambda_{0}^{*}=\lambda_{1}^{*}=\lambda_{2}^{*}=\frac{1}{16}$. Thus, in view of Theorem 3.3, problem (3.37) has at least one solution on $\mathcal{J}$.

In addition, for $u_{1}, u_{2}, u_{3}, \bar{u}_{1}, \bar{u}_{2}, \bar{u}_{1} \in \mathbb{R}_{+}$and $t \in \mathcal{J}$,

$$
\left|f\left(t, u_{1}, u_{2}, u_{3}\right)-f\left(t, \bar{u}_{1}, \bar{u}_{2}, \bar{u}_{3}\right)\right| \leq \frac{1}{16}\left(\left|u_{1}-\bar{u}_{1}\right|+\left|u_{2}-\bar{u}_{2}\right|+\left|u_{3}-\bar{u}_{3}\right|\right) .
$$

Hence, $\left(\mathcal{A}_{4}\right)$ is satisfied with $\mathcal{K}_{1}=\mathcal{K}_{2}=\frac{1}{16}$. Let us denote $\Phi(t)=t+1$. Obviously, $\Phi$ is an increasing function on $[0,1]$ and $\Phi^{\prime}(t)=1$ is a continuous on $[0,1]$. Furthermore, by simple computation, we get $\Psi_{1} \approx 1.5628>0$ and

$$
\left(\frac{2 \mathcal{K}_{1}}{1-\mathcal{K}_{2}}\right) \Psi_{1} \approx 0.2684<1
$$

which implies that hypothesis $\left(\mathcal{A}_{5}\right)$ is satisfied. Hence, it follows from Theorem 3.4 that problem (3.37) has a unique solution on $\mathcal{J}$.

Example 3.18 Let IFPBVPs be described by

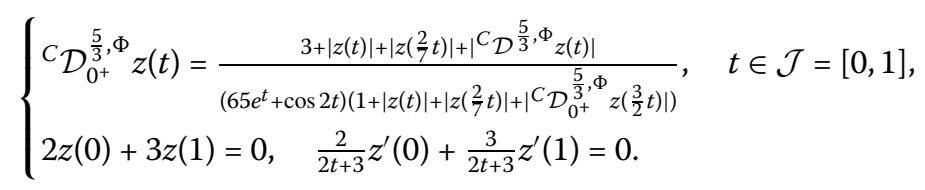

By comparing (3.38) with (1.5), we get $r=\frac{5}{3}, \alpha_{1}=2, \alpha_{2}=3, \sigma=\frac{2}{7}, \xi=1$ and let us denote $\Phi(t)=t^{2}+3 t+1$. This implies that $\Phi(t)$ is an increasing function on $[0,1]$ and $\Phi^{\prime}(t)=$ $2 t+3 \neq 0$, is continuous for all $t \in[0,1]$. Moreover, the function $f: \mathcal{J} \times \mathbb{R}^{3} \rightarrow \mathbb{R}$ defined by

$$
f\left(t, u_{1}, u_{2}, u_{3}\right)=\frac{3+\left|u_{1}\right|+\left|u_{2}\right|+\left|u_{3}\right|}{\left(65 e^{t}+\cos 2 t\right)\left(1+\left|u_{1}\right|+\left|u_{2}\right|+\left|u_{3}\right|\right)}, \quad t \in \mathcal{J}, u_{1}, u_{2}, u_{3} \in \mathbb{R}_{+},
$$


is continuous and

$$
\left|f\left(t, u_{1}, u_{2}, u_{3}\right)\right| \leq \frac{1}{\left(65 e^{t}+\cos 2 t\right)}\left(3+\left|u_{1}\right|+\left|u_{2}\right|+\left|u_{3}\right|\right), \quad t \in \mathcal{J},
$$

which implies that $\left(\mathcal{A}_{3}\right)$ is satisfied with $\lambda_{0}(t)=\frac{3}{\left(65 e^{t}+\cos 2 t\right)}, \lambda_{1}(t)=\lambda_{2}(t)=\frac{1}{\left(65 e^{t}+\cos 2 t\right)}$ and $\lambda_{0}^{*}=\frac{3}{66}, \lambda_{1}^{*}=\lambda_{2}^{*}=\frac{1}{66}$. Hence, problem (3.38) has at least one solution on $\mathcal{J}$, since all the assumptions of Theorem 3.11 hold.

In addition, for all $u_{1}, u_{2}, u_{3}, \bar{u}_{1}, \bar{u}_{2}, \bar{u}_{3} \in \mathbb{R}_{+}$and $t \in \mathcal{J}$,

$$
\left|f\left(t, u_{1}, u_{2}, u_{3}\right)-f\left(t, \bar{u}_{1}, \bar{u}_{2}, \bar{u}_{3}\right)\right| \leq \frac{1}{66}\left(\left|u_{1}-\bar{u}_{1}\right|+\left|u_{2}-\bar{u}_{2}\right|+\left|u_{3}-\bar{u}_{3}\right|\right) .
$$

Hence, hypothesis $\left(\mathcal{A}_{4}\right)$ is satisfied with $\mathcal{K}_{1}=\mathcal{K}_{2}=\frac{1}{66}$. Thus, by simplification this gives $\Psi_{2} \approx 14.7379>0$ and

$$
\left(\frac{2 \mathcal{K}_{1}}{1-\mathcal{K}_{2}}\right) \Psi_{2} \approx 0.4535<1
$$

Therefore, as a result of Theorem 3.12, problem (3.38) has a unique solution on $\mathcal{J}$.

\section{Conclusions}

Nonlinear analysis is one of the best methods to analyze the applied problems. In this paper, we were able to obtain an equivalent integral equation by formulating the Green's functions of the proposed problems (1.4) and (1.5). With the help of Schaefer's and Banach's fixed point theorems, we investigated the existence and uniqueness of solutions to the proposed problems (1.4) and (1.5). In addition:

- The results obtained in this paper generalized those obtained from the work of [1-3, 8, 14, 33], (see, respectively, Corollaries 3.5, 3.6, 3.13 and 3.14).

- For $\Phi(t)=t$ and taking $\alpha_{1} \neq \alpha_{2}>1$, we constructed the Green's function of problems (1.4)-(1.5) and the existence and uniqueness of solutions were presented, (see respectively, Corollaries 3.7, 3.8, 3.15 and 3.16).

In this context, we conclude that the results obtained in this paper are new and concern results widespread in the literature and this achievement can be regarded as a contribution to the improvement of the qualitative aspect of fractional calculus. An interesting extension of the proposed problems (1.4) and (1.5) would be to investigate the positive solutions and stability analysis.

\section{Acknowledgements}

The authors acknowledge the financial support provided by the Center of Excellence in Theoretical and Computational Science (TaCS-CoE), KMUTT. The first and the sixth authors were supported by "Petchra Pra Jom Klao Ph.D. Research Scholarship from King Mongkut's University of Technology Thonburi" (Grant No. 13/2561). Furthermore, Wiyada Kumam was financial supported by the Rajamangala University of Technology Thanyaburi (RMUTTT) (Grant No. NSF62D0604). Moreover, the authors express their gratitude for the positive comments received from anonymous reviewers and the editors, which have improved the readability and correctness of the paper.

\section{Funding}

Petchra Pra Jom Klao Doctoral Scholarship for Ph.D. program of King Mongkut's University of Technology Thonburi (KMUTT). The Center of Excellence in Theoretical and Computational Science (TaCS-CoE), KMUTT. Rajamangala University of Technology Thanyaburi (RMUTTT) (Grant No. NSF62D0604). 
Availability of data and materials

Not applicable.

\section{Competing interests}

The authors declare that they have no competing interests.

\section{Authors' contributions}

The authors contributed equally to this paper. All authors have read and approved the final version of the manuscript.

\section{Author details}

'Department of Mathematics, Faculty of Science, King Mongkut's University of Technology Thonburi (KMUTT), 126 Pracha-Uthit Road, Bang Mod, Thung Khru, Bangkok 10140, Thailand. ${ }^{2}$ Fixed Point Research Laboratory, Fixed Point Theory and Applications Research Group, Center of Excellence in Theoretical and Computational Science (TaCS-CoE), Faculty of Science, King Mongkut's University of Technology Thonburi (KMUTT), 126 Pracha-Uthit Road, Bang Mod, Thung Khru, Bangkok 10140, Thailand. ${ }^{3}$ Department of Mathematics and General Sciences, Prince Sultan University, Riyadh, Saudi Arabia. ${ }^{4}$ Department of Mathematics, Faculty of Arts and Sciences, Çankaya University, Ankara, 06790, Turkey. ${ }^{5}$ Department of Medical Research, China Medical University Hospital, China Medical University, Taichung 40402, Taiwan. ${ }^{6}$ Department of Computer Science and Information Engineering, Asia University Hospital, Taichung 40402, Taiwan. ${ }^{7}$ Program in Applied Statistics, Department of Mathematics and Computer Science, Rajamangala University of Technology Thanyaburi, Thanyaburi, Pathumthani 12110, Thailand.

\section{Publisher's Note}

Springer Nature remains neutral with regard to jurisdictional claims in published maps and institutional affiliations.

Received: 27 May 2020 Accepted: 24 September 2020 Published online: 06 October 2020

\section{References}

1. Agarwal, R.P., Ahmad, B., Alsaedi, A.: Fractional-order differential equations with anti-periodic boundary conditions: a survey. Bound. Value Probl. 2017(1), 1 (2017)

2. Ahmad, B.: Existence of solutions for fractional differential equations of order $q \in(2,3]$ with anti-periodic boundary conditions. J. Appl. Math. Comput. 34(1-2), 385-391 (2010)

3. Ahmad, B., Nieto, J.J., et al.: Existence of solutions for anti-periodic boundary value problems involving fractiona differential equations via Leray-Schauder degree theory. Topol. Methods Nonlinear Anal. 35(2), 295-304 (2010)

4. Ahmad, B., Otero-Espinar, V.: Existence of solutions for fractional differential inclusions with antiperiodic boundary conditions. Bound. Value Probl. 2009, 1 (2009)

5. Ahmed, I., Kumam, P., Jarad, F., Borisut, P., Jirakitpuwapat, W.: On Hilfer generalized proportional fractional derivative. Adv. Differ. Equ. 2020(1), 1 (2020)

6. Ahmed, I., Kumam, P., Jarad, F., Borisut, P., Sitthithakerngkiet, K., Ibrahim, A.: Stability analysis for boundary value problems with generalized nonlocal condition via Hilfer-Katugampola fractional derivative. Adv. Differ. Equ. 2020(1), 1 (2020)

7. Ahmed, I., Kumam, P., Shah, K., Borisut, P., Sitthithakerngkiet, K., Demba, M.A.: Stability results for implicit fractional pantograph differential equations via $\phi$-Hilfer fractional derivative with a nonlocal Riemann-Liouville fractional integral condition. Mathematics 8(1), 94 (2020)

8. Ali, A., Shah, K., Abdeljawad, T.: Study of implicit delay fractional differential equations under anti-periodic boundary conditions. Adv. Differ. Equ. 2020, 139 (2020)

9. Almeida, R.: A Caputo fractional derivative of a function with respect to another function. Commun. Nonlinear Sci. Numer. Simul. 44, 460-481 (2017)

10. Atangana, A.: Derivative with a New Parameter: Theory, Methods and Applications. Academic Press, San Diego (2015)

11. Atangana, A.: Fractional Operators with Constant and Variable Order with Application to Geo-Hydrology. Academic Press, San Diego (2017)

12. Atangana, A.: Modelling the spread of COVID-19 with new fractal-fractional operators: can the lockdown save mankind before vaccination? Chaos Solitons Fractals 136, 109860 (2020)

13. Balachandran, K., Kiruthika, S., Trujillo, J.: Existence of solutions of nonlinear fractional pantograph equations. Acta Math. Sci. 33(3), 712-720 (2013)

14. Benchohra, M., Hamidi, N., Henderson, J.: Fractional differential equations with anti-periodic boundary conditions. Numer. Funct. Anal. Optim. 34(4), 404-414 (2013)

15. Bhalekar, S., Patade, J.: Series solution of the pantograph equation and its properties. Fractal Fract. 1(1), 16 (2017)

16. Borisut, P., Kumam, P., Ahmed, I., Sitthithakerngkiet, K.: Nonlinear Caputo fractional derivative with nonlocal Riemann-Liouville fractional integral condition via fixed point theorems. Symmetry 11(6), 829 (2019)

17. Gambo, Y.Y., Jarad, F., Baleanu, D., Abdeljawad, T.: On Caputo modification of the Hadamard fractional derivatives. Adv. Differ. Equ. 2014, 10 (2014)

18. Harikrishnan, S., Shah, K., Kanagarajan, K.: Existence theory of fractional coupled differential equations via $\psi$-Hilfer fractional derivative. Random Oper. Stoch. Equ. 27(4), 207-212 (2019)

19. Jarad, F., Abdeljawad, T.: Generalized fractional derivatives and Laplace transform. Discrete Contin. Dyn. Syst., Ser. S 13(3), 709-722 (2019)

20. Jarad, F., Abdeljawad, T., Baleanu, D.: On the generalized fractional derivatives and their Caputo modification. J. Nonlinear Sci. Appl. 10(5), 2607-2619 (2017)

21. Kilbas, A., Srivastava, H., Trujillo, J.: Theory and Applications of Fractional Derivatial Equations. North-Holland Mathematics Studies, vol. 204. Elsevier, New York (2006)

22. Li, X., Chen, F., Li, X.: Generalized anti-periodic boundary value problems of impulsive fractional differential equations. Commun. Nonlinear Sci. Numer. Simul. 18(1), 28-41 (2013) 
23. Liu, M., Li, D.: Properties of analytic solution and numerical solution of multi-pantograph equation. Appl. Math. Comput. 155(3), 853-871 (2004)

24. Luchko, Y., Trujillo, J.: Caputo-type modification of the Erdélyi-Kober fractional derivative. Fract. Calc. Appl. Anal. 10(3), 249-267 (2007)

25. Mainardi, F.: Fractional Calculus and Waves in Linear Viscoelasticity: An Introduction to Mathematical Models. World Scientific, Singapore (2010)

26. Nieto, J., Ouahab, A., Venktesh, V.: Implicit fractional differential equations via the Liouville-Caputo derivative. Mathematics 3(2), 398-411 (2015)

27. Podlubny, I.: Fractional Differential Equations: An Introduction to Fractional Derivatives, Fractional Differential Equations, to Methods of Their Solution and Some of Their Applications, vol. 198. Elsevier, Amsterdam (1998)

28. Samet, B., Aydi, H.: Lyapunov-type inequalities for an anti-periodic fractional boundary value problem involving $\psi$-Caputo fractional derivative. J. Inequal. Appl. 2018, 286 (2018)

29. Samko, S.G., Kilbas, A.A., Marichev, O.I, et al.: Fractional Integrals and Derivatives, vol. 1993. Gordon \& Breach, Yverdon-les-Bains (1993)

30. Shah, K., Sarwar, M., Baleanu, D., et al.: Study on Krasnoselskii's fixed point theorem for Caputo-Fabrizio fractional differential equations. Adv. Differ. Equ. 2020(1), 1 (2020)

31. Vivek, D., Kanagarajan, K., Elsayed, E.: Some existence and stability results for Hilfer-fractional implicit differential equations with nonlocal conditions. Mediterr. J. Math. 15(1), 15 (2018)

32. Wang, J., Zada, A., Waheed, H.: Stability analysis of a coupled system of nonlinear implicit fractional anti-periodic boundary value problem. Math. Methods Appl. Sci. 42(18), 6706-6732 (2019)

33. Wang, K., Li, Y.: A note on existence of (anti-) periodic and heteroclinic solutions for a class of second-order odes. Nonlinear Anal., Theory Methods Appl. 70(4), 1711-1724 (2009)

34. Yang, D., Bai, C.: Existence of solutions for anti-periodic fractional differential inclusions with caupto fractional derivative. Discrete Dyn. Nat. Soc. 2019, Article ID 9824623 (2019)

35. Zada, A., Waheed, H.: Stability analysis of implicit fractional differential equation with anti-periodic integral boundary value problem. Ann. Univ. Paedagog. Crac. Stud. Math. 1(19), 5-25 (2020)

36. Zhou, Y., Wang, J., Zhang, L.: Basic Theory of Fractional Differential Equations. World Scientific, Singapore (2016)

\section{Submit your manuscript to a SpringerOpen ${ }^{0}$ journal and benefit from:}

- Convenient online submission

- Rigorous peer review

- Open access: articles freely available online

- High visibility within the field

- Retaining the copyright to your article

Submit your next manuscript at $\gg$ springeropen.com 